\title{
Symmetry of anisotropic exchange interactions in semiconductor nanostructures
}

\author{
K.V.Kavokin \\ School of Physics, University of Exeter, Stocker Road, Exeter EX4 4QL, \\ UK, and A. F. Ioffe Physico-Technical Institute, 194021 \\ Politechnicheskaya 26, \\ St. Petersburg, Russia
}

\begin{abstract}
The symmetry of exchange interaction of charge carriers in semiconductor nanostructures (quantum wells and quantum dots) is analysed. It is shown that the exchange Hamiltonian of two particles belonging to the same energy band can be universally expressed via pseudospin operators of the particles. The relative strength of the anisotropic exchange interaction is shown to be independent of the binding energy and the isotropic exchange constant.
\end{abstract}

The reduced symmetry of semiconductor nanostructures suggests that the exchange interaction of charge carriers in such structures is not necessarily described by the isotropic (Heisenberg) spin Hamiltonian. In particular, the exchange interaction of electrons and holes in quantum wells and quantum dots is known to be extremely anisotropic, giving rise to a fine structure of nanostructure excitons [1]. It has been shown [2] that the exchange interaction of conduction-band electrons is also anisotropic if the structure lacks inversion symmetry. The main term of the anisotropic exchange Hamiltonian in this case has the Dzyaloshinskii-Moriya form [3]. The electron-electron anisotropic exchange was subsequently widely discussed in relation to the quantum computing problem [4.5.6.6]. It has been recently detected experimentally via its contribution to the spin relaxation of donorbound electrons in GaAs [7], where it has been shown to put the upper boundary for the 
electron spin lifetime at donor concentrations around $10^{16} \mathrm{~cm}^{-3}$. However, it remained so far unclear whether or not the anisotropic spin Hamiltonian suggested in Ref. [2] is universal for all types of charge carriers, e.g. for two-dimensional holes. The issue of the dependence of the anisotropic exchange constant on the parameters of the localizing potential is also very sensitive, especially for the discussion on feasibility of quantum computation with solidstate spin systems [5]. The constant was so far calculated using the Heitler-London method [2.5], which is known to give incorrect asymptotic expression for the isotropic exchange integral [8,9]. Gor'kov and Krotkov [10], using the median-plane method [8], have recently obtained a correct asymptotic formula for the anisotropic exchange constant in a specific case of hydrogen-like centers in zinc-blende semiconductors, different from that calculated earlier by the Heitler-London method [2]. However their approach is not always applicable to coupled quantum dots, where the distance between quantum dots can be comparable to the quantum-dot size.

The collection of unsolved problems and blanc spaces in the existing knowledge on the anisotropic exchange in semiconductor structures, given above, demonstrates the evident demand for a consistent theoretical analysis of the issue, based on a general approach. In this paper, we consider exchange interaction of two identical charge carriers localized in any symmetric double-well potential in a two-dimensional semiconductor structure. Using the pseudospin formalism allows to obtain a universal spin Hamiltonian describing this class of systems.

Let us consider the exchange interaction of two identical charge carriers (electrons or holes), localized in two centrosymmetric potential hollows (further referred to as quantum dots, QDs) in a quasi-two-dimensional semiconductor structure (quantum well, QW). The QDs may be, for example, self-organized QDs [11]; otherwise, they can be induced by electrostatic potential of nanometer-sized gates [12] or impurity centers [13]. The distance between centers of the QDs will further be denoted as $R_{12}$. In quasi-two-dimensional structures, the 4-fold degeneration of the valence band, typical of cubic semiconductors, is lifted. The states at extremum points of two-dimensional subbands in absence of magnetic fields retain only 
the Kramers two-fold degeneration. Their wave functions can be written as $\Psi(\mathbf{r}) u_{\nu}(\mathbf{r})$, where is an envelope function, and $u_{\nu}(\mathbf{r})$ is a Bloch amplitude, $\nu= \pm 1 / 2$. The Bloch amplitudes $u_{\nu}(\mathbf{r})$ are transformed into each other by the operator of time reversal [14]:

$$
u_{-1 / 2}(\mathbf{r})=-i \hat{\sigma}_{y} u_{+1 / 2}(\mathbf{r})
$$

This property allows to associate the Kramers index $\nu$ with an eigenvalue of a projection of a pseudospin operator $\mathbf{j}(j=1 / 2)$ on some (generally, fictitious) axis. The choice of basis functions for $\mathbf{j}$ is not unambiguous. It is limited only by the condition given by Eq.(1). In particular, for heavy holes with the projection of the angular momentum on the structure axis Z, equal to $J_{z}= \pm 3 / 2$, it is convenient to choose the functions as [1]: $|j,+1 / 2\rangle=\left|J_{z},-3 / 2\right\rangle$ and $|j,-1 / 2\rangle=\left|J_{z},+3 / 2\right\rangle$. This choice allows to avoid phase multipliers which would otherwise appear at wave functions in the pseudospin representation. For conduction-band electrons, the pseudospin coincides with the electron spin s. Linear transformations of pseudospin wave functions determined in the basis $\left\{u_{+1 / 2}, u_{-1 / 2}\right\}$ are equivalent to rotations of usual spinor functions [14:

$$
\begin{aligned}
& u_{+1 / 2}^{\alpha \beta \gamma}=\exp (i \gamma / 2)\left[u_{+1 / 2} \exp (i \alpha / 2) \cos (\beta / 2)+u_{-1 / 2} \exp (-i \alpha / 2) \sin (\beta / 2)\right] \\
& u_{-1 / 2}^{\alpha \beta \gamma}=\exp (-i \gamma / 2)\left[-u_{+1 / 2} \exp (i \alpha / 2) \sin (\beta / 2)+u_{-1 / 2} \exp (-i \alpha / 2) \cos (\beta / 2)\right]
\end{aligned}
$$

where $\alpha, \beta$, and $\gamma$ are analogs of Euler angles. Following the analogy, one can introduce the total pseudospin $\mathbf{I}=\mathbf{j}_{1}+\mathbf{j}_{2}$. Indeed, the Gilbert space of two-pseudospin wave functions $A_{\mu \nu} u_{\mu}\left(\mathbf{r}_{1}\right) u_{\nu}\left(\mathbf{r}_{2}\right)$ breaks into two subspaces invariant with respect to the simultaneous transformation of both pseudospins along Eq.(2) with the same $\alpha, \beta$, and $\gamma$. The basis functions of these subspaces, $\xi_{0}=\left(u_{+1 / 2}\left(\mathbf{r}_{1}\right) u_{-1 / 2}\left(\mathbf{r}_{2}\right)-u_{-1 / 2}\left(\mathbf{r}_{1}\right) u_{+1 / 2}\left(\mathbf{r}_{2}\right)\right) / \sqrt{2}$ and $\xi_{1 M}$, equal to $\left(u_{+1 / 2}\left(\mathbf{r}_{1}\right) u_{-1 / 2}\left(\mathbf{r}_{2}\right)+u_{+1 / 2}\left(\mathbf{r}_{1}\right) u_{-1 / 2}\left(\mathbf{r}_{2}\right)\right) / \sqrt{2}(M=0)$ or $u_{ \pm 1 / 2}\left(\mathbf{r}_{1}\right) u_{ \pm 1 / 2}\left(\mathbf{r}_{2}\right)(M= \pm 1)$, are, obviously, eigenfunctions of the operators $\hat{I}^{2}$ and $\hat{I}_{z}$.

The general form of the one-particle Hamiltonian of the two dimensional charge carrier in the pseudospin representation is

$$
\hat{H}_{1}=\frac{\hbar^{2}}{2 m} \hat{k}^{2}+V(\mathbf{r})+\mathbf{h}(\mathbf{k}) \cdot \mathbf{j}
$$


where the "spin-orbit field" $\mathbf{h}(\mathbf{k})$ is a vector in the pseudospin space [15]. $\mathbf{h}(\mathbf{k})$ is an odd function of the components of the wave vector. It is not equal to zero if the structure lacks inversion symmetry (which is very typical for nanostructures). This is the case when either the crystal unit cell lacks inversion symmetry (bulk inversion asymmetry, BIA [16]), or the QW is asymmetric (structure inversion asymmetry, SIA [17]). The components of $\mathbf{h}(\mathbf{k})$ may be, or may not be, associated with certain Cartesian axes in the real space. In the two-dimensional case, $\mathbf{h}(\mathbf{k})$ is dominated by linear in $\mathbf{k}$ terms 17, 18, 19:

$$
h_{\eta}=A_{\eta \zeta} k_{\zeta}
$$

where the matrix $A$ is defined by the structure symmetry.

The problem we are going to solve is finding the fine structure of the ground state of the two-particle Hamiltonian:

$$
\hat{H}=\hat{H}_{0}+\hat{H}_{S O}
$$

where

$$
\begin{gathered}
\hat{H}_{0}=\frac{\hbar^{2}}{2 m} \hat{k}_{1}^{2}+\frac{\hbar^{2}}{2 m} \hat{k}_{2}^{2}+V_{1}\left(\mathbf{r}_{1}\right)+V_{2}\left(\mathbf{r}_{2}\right)+U_{12}\left(\left|\mathbf{r}_{1}-\mathbf{r}_{2}\right|\right) \\
\hat{H}_{S O}=\mathbf{h}\left(\mathbf{k}_{1}\right) \cdot \mathbf{j}_{1}+\mathbf{h}\left(\mathbf{k}_{2}\right) \cdot \mathbf{j}_{2}
\end{gathered}
$$

and $U_{12}\left(\left|\mathbf{r}_{1}-\mathbf{r}_{2}\right|\right)$ is the operator of the Coulomb interaction between the two particles. Before tackling the effects of spin-orbit interaction in the form of Eq.(7) on the exchange interaction, we should reconsider the ground-state structure of the Hamiltonian $H_{0}$. It is indeed well-known for electrons whose one-particle wave functions are $\Psi(\mathbf{r}) \zeta_{\mu}$, where the spinor $\zeta_{\mu}$ is not a function of coordinates. To the contrary, the Bloch amplitude $u_{\nu}$ does depend on coordinates, and, moreover, it may contain spinors with both $\mu=+1 / 2$ and $\mu=-1 / 2$. The exciton (an electron-hole pair) in a QD is a good example demonstrating that the exchange interaction of charge carriers may have a very different symmetry as compared to that of free electrons. The QD exciton fine structure [1] consists of two doublets, being 
thus quite different from the fine structure of a pair of vacuum electrons, i.e. the well-known singlet-triplet structure associated with the Heisenberg exchange.

In order to analyze the fine structure of $H_{0}$ for two particles belonging to the same subband, we first note that their behavior should be identical to that of bare electrons in all aspects but the Coulomb interaction. Indeed, although Bloch amplitudes are functions of coordinates within the unit cell, the one-particle operators of the kinetic energy and of the potential energy in Eq.(6) in the effective-mass approximation act upon envelope function, not Bloch amplitudes. Therefore, with respect to these one-particle operators, the Bloch amplitudes are just equivalent to spinors. To the contrary, calculating the Coulomb energy assumes taking integrals over the unit sell also. It is due this fact that the symmetry of Bloch amplitudes of holes and electrons has an impact on the fine structure in the exciton 11.20".

The fermionic wave functions of the two charge carriers can be written in the following form, similar to that of bare electrons:

$$
\begin{gathered}
\Psi_{0}\left(\mathbf{r}_{1}, \mathbf{r}_{2}\right)=\left[\Phi_{0}\left(\mathbf{r}_{1}, \mathbf{r}_{2}\right)+\Phi_{0}\left(\mathbf{r}_{2}, \mathbf{r}_{1}\right)\right] \xi_{0} \\
\Psi_{1 M}\left(\mathbf{r}_{1}, \mathbf{r}_{2}\right)=\left[\Phi_{1}\left(\mathbf{r}_{1}, \mathbf{r}_{2}\right)-\Phi_{1}\left(\mathbf{r}_{2}, \mathbf{r}_{1}\right)\right] \xi_{1 M}
\end{gathered}
$$

where $\Phi_{0}\left(\mathbf{r}_{1}, \mathbf{r}_{2}\right)$ and $\Phi_{1}\left(\mathbf{r}_{1}, \mathbf{r}_{2}\right)$ are two-particle envelope functions defined so that each particle is most likely to be found near its "home" center, while $\Phi_{0}\left(\mathbf{r}_{2}, \mathbf{r}_{1}\right)$ and $\Phi_{1}\left(\mathbf{r}_{2}, \mathbf{r}_{1}\right)$ correspond to interchanged particle positions. To determine the structure of respective energy levels, we should recall a property of the Bloch amplitudes $u_{\nu}$, which results from their symmetry with respect to time reversal, and is an equivalent formulation of the Kramers theorem. As it follows from the Kramers theorem, the states symmetric with respect to time reversal remain degenerated unless magnetic field is applied. Mathematically, this means that matrix elements of any function of coordinates (not containing derivatives or spin operators) between $u_{+1 / 2}(\mathbf{r})$ and $u_{-1 / 2}(\mathbf{r})$ are zero, while diagonal matrix elements are equal to each other: 


$$
\begin{aligned}
& \int_{\Omega} u_{+1 / 2}(\mathbf{r}) u_{-1 / 2}^{*}(\mathbf{r}) f(\mathbf{r}) d^{3} r=\int_{\Omega} u_{-1 / 2}(\mathbf{r}) u_{+1 / 2}^{*}(\mathbf{r}) f(\mathbf{r}) d^{3} r=0 \\
& \int_{\Omega} u_{+1 / 2}(\mathbf{r}) u_{+1 / 2}^{*}(\mathbf{r}) f(\mathbf{r}) d^{3} r=\int_{\Omega} u_{-1 / 2}(\mathbf{r}) u_{-1 / 2}^{*}(\mathbf{r}) f(\mathbf{r}) d^{3} r
\end{aligned}
$$

where the integrals are taken over the unit cell.

Using Eq.(9), one can easily find that

$$
\begin{aligned}
\left\langle\Psi_{0}\left(\mathbf{r}_{1}, \mathbf{r}_{2}\right)\left|U_{12}\left(\left|\mathbf{r}_{1}-\mathbf{r}_{2}\right|\right)\right| \Psi_{1 M}\left(\mathbf{r}_{1}, \mathbf{r}_{2}\right)\right\rangle & =0 \\
\left\langle\Psi_{0}\left(\mathbf{r}_{1}, \mathbf{r}_{2}\right)\left|U_{12}\left(\left|\mathbf{r}_{1}-\mathbf{r}_{2}\right|\right)\right| \Psi_{0}\left(\mathbf{r}_{1}, \mathbf{r}_{2}\right)\right\rangle & \neq \\
\left\langle\Psi_{1 M}\left(\mathbf{r}_{1}, \mathbf{r}_{2}\right)\left|U_{12}\left(\left|\mathbf{r}_{1}-\mathbf{r}_{2}\right|\right)\right| \Psi_{1 M^{\prime}}\left(\mathbf{r}_{1}, \mathbf{r}_{2}\right)\right\rangle & =\text { const } \cdot \delta_{M M^{\prime}}
\end{aligned}
$$

Thus, the Coulomb interaction retains the singlet-triplet structure of the ground state of two identical charge carriers. Exactly like in the case of two bare electrons, two-particle states with the same total pseudospin $I$ are degenerated. Consequently, the Hamiltonian of the exchange interaction in terms of pseudospin operators takes the Heisenberg form:

$$
\hat{H}_{S}=-2 \Delta\left(\mathbf{j}_{1} \cdot \mathbf{j}_{2}+1\right)
$$

where $\Delta$ is a constant to be determined for each specific case.

Now we can consider the effect of the spin-orbit terms given by the Eq.(7) on the exchange interaction. In the following, we will choose the axis $\mathrm{X}$ along the straight line connecting the localization centers (QDs). To handle the spin-orbit terms, we make use of a unitary transformation proposed by Levitov and Rashba [21] who used it to eliminate spin-orbit terms in the one-dimensional case. The matrix $T$ defined as

$$
T=\exp \left[i \frac{2 m}{\hbar^{2}} \sum_{\alpha} A_{\alpha x}\left(\hat{\jmath}_{1 \alpha} x_{1}+\hat{\jmath}_{2 \alpha} x_{2}\right)\right]
$$

transforms the Hamiltonian Eq.(5) into the form:

$$
T \hat{H} T^{-1}=\hat{H}^{\prime}=\hat{H}_{0}^{\prime}+\hat{H}_{S O}^{\prime}
$$

where 


$$
\hat{H}_{0}^{\prime}=\frac{\hbar^{2}}{2 m} \hat{k}_{1}^{\prime 2}+\frac{\hbar^{2}}{2 m} \hat{k}_{2}^{\prime 2}+V_{1}\left(\mathbf{r}_{1}^{\prime}\right)+V_{2}\left(\mathbf{r}_{2}^{\prime}\right)+U_{12}\left(\left|\mathbf{r}_{1}^{\prime}-\mathbf{r}_{2}^{\prime}\right|\right)-\sum_{\alpha} \frac{m\left(A_{\alpha x}\right)^{2}}{\hbar^{2}}
$$

and

$$
\hat{H}_{S O}^{\prime}=\sum_{\alpha} A_{\alpha y}\left(k_{1 y} \hat{\jmath}_{1 \alpha}^{\prime}+k_{2 y} \hat{\jmath}_{2 \alpha}^{\prime}\right)
$$

where $\mathbf{j}_{1}^{\prime}=T \mathbf{j}_{1} T^{-1}, \mathbf{j}_{2}^{\prime}=T \mathbf{j}_{2} T^{-1}$.

The Hamiltonian $\hat{H}^{\prime}$ does not contain spin-orbit terms and therefore results in the exchange interaction in the form of Eq.(11):

$$
\hat{H}_{S}^{\prime}=-2 \Delta\left(\mathbf{j}_{1}^{\prime} \cdot \mathbf{j}_{2}^{\prime}+1\right)
$$

Due to the axial symmetry of the system, the matrix elements of $k_{1 y}$ and $k_{2 y}$, calculated on the ground-state eigenfunctions of $\hat{H}_{0}^{\prime}$ (they can be obtained from Eq.(8) by the transformation with the matrix $T$, which does not affect their dependence on $y$ ), are exactly equal to zero. The same is true for all the odd powers of $k_{1 y}$ and $k_{2 y}$. Therefore, $\hat{H}_{S O}^{\prime}$ does not contribute into the exchange interaction.

Finally, to obtain the exchange Hamiltonian in the non-transformed basis, one should substitute the expressions for $\mathbf{j}_{1}^{\prime}$ and $\mathbf{j}_{2}^{\prime}$ into Eq.(16). Since the transformation $T$ is a rotation through the angle $\frac{2 m}{\hbar^{2}} \sqrt{\sum_{\nu} A_{\nu x} A_{\nu x}} x$ around the vector $A_{\nu x}$ in the pseudospin space, $\hat{H}_{S}$ in the non-transformed basis is not unambiguously defined: it depends on the coordinates $x_{1}$ and $x_{2}$ at which we take the spin operators $\mathbf{j}_{1}$ and $\mathbf{j}_{2}$. A natural choice is to define them at the centers of corresponding QDs; for instance, this definition allows to write the Zeeman interaction in the usual form, $\hat{H}_{Z}=\mu_{B} g_{\alpha \beta} j_{\alpha} B_{\beta}$, where $B$ is the magnetic field, $\mu_{B}$ is the Bohr magneton, and $g_{\alpha \beta}$ is a symmetric tensor g-factor [22 whose principal directions do not depend on the envelope wave function of the localized particle. This way, we come to the expression for $\hat{H}_{S}$ obtained in Ref. [2]:

$$
\hat{H}_{S}=-2 \Delta\left(1+\mathbf{j}_{1} \cdot \mathbf{j}_{2} \cos \gamma+\left(\mathbf{d} \cdot \mathbf{j}_{\mathbf{1}}\right)\left(\mathbf{d} \cdot \mathbf{j}_{\mathbf{2}}\right)(\mathbf{1}-\cos \gamma)+\mathbf{d} \cdot\left[\mathbf{j}_{1} \times \mathbf{j}_{2}\right] \sin \gamma\right)
$$

where $\gamma=\frac{2 m}{\hbar^{2}} \sqrt{\sum_{\nu} A_{\nu x} A_{\nu x}} R_{12}$, and $\mathbf{d}$ is a unit vector in the pseudospin space, defined so that $d_{\nu}=A_{\nu x} / \sqrt{\sum_{\nu} A_{\nu x} A_{\nu x}}$. The first anisotropic term has the form of pseudodipole 
interaction [22], and the second one, of the Dzyaloshinskii-Moriya interaction [3]. At small $\gamma$, the Dzyaloshinskii-Moriya interaction dominates.

The Eq.(17) demonstrates a remarkable universality of the exchange interaction in twodimensional semiconductor nanostructures: this form of the Hamiltonian holds for both electrons and holes, for any type of centrosymmetric localizing potentials. The Eq.(17) is valid for identical as well as for different QDs. Moreover, the angle $\gamma$ characterizing the relative strength of the anisotropic exchange depends only on the distance between the QDs and the orientation of the pair of QDs with respect to the crystal axes. It is not sensitive to binding energies of the charge carriers in the QDs and to the value of the isotropic exchange constant $\Delta$.

The value of $\gamma$ can be now easily calculated for those structures where the components of the matrix $A$ are known.

In [100] oriented GaAs quantum wells the dominating BIA terms are 19 $A_{y y}=-A_{x x}=$ $\frac{\alpha \hbar^{3}}{m \sqrt{2 m E_{g}}}\left\langle k_{z}^{2}\right\rangle, A_{x y}=A_{y x}=0$ where $\alpha \approx 0.65$ [23] (here coordinates $x$ and $y$ are taken along the cubic crystal axes). This gives $\gamma=\frac{2 \alpha \hbar}{\sqrt{2 m E_{g}}}\left\langle k_{z}^{2}\right\rangle R_{12}$. For example, in a 5nm-wide QW considered in Ref. [5], $\gamma=\left(3 \cdot 10^{5} \mathrm{~cm}^{-1}\right) R_{12}$, which gives for $R=24 \mathrm{~nm} \gamma=0.72$, i.e. more than an order of magnitude greater than the value of 0.02 obtained in Ref. [5] at the same values of parameters by the Heitler-London method.

For Rashba terms, $A_{y y}=A_{x x}=0, A_{x y}=-A_{y x}=a$. In a single-side modulation-doped n-type $\mathrm{Si} / \mathrm{SiGe}$ quantum well, the constant $a$ of $1.1 \cdot 10^{-12} \mathrm{eV} \cdot \mathrm{cm}$ was measured [24]. This gives $\gamma=\frac{2 m a}{\hbar^{2}} R_{12} \approx\left(6.7 \cdot 10^{2} \mathrm{~cm}^{-1}\right) R_{12}$.

Bulk inversion asymmetry terms for holes in zinc-blende semiconductors include both cubic and linear in $k$ terms [25]. The cubic term $H_{3 V}$ has the same symmetry as the Dresselhaus term for electrons, with the constant $\frac{\alpha_{V} \hbar^{3}}{m \sqrt{2 m E_{g}}}$, where $m$ is the conduction-band electron mass, and $\alpha_{V} \approx 0.1$ for GaAs. The linear term is given by the expression:

$$
H_{1 V}=\frac{4}{\sqrt{3}} \varkappa(\mathbf{k} \cdot \Omega)
$$

where $\Omega_{z}=\hat{J}_{z}\left(\hat{J}_{x}^{2}-\hat{J}_{y}^{2}\right)+\left(\hat{J}_{x}^{2}-\hat{J}_{y}^{2}\right) \hat{J}_{z}$ (other components of $\boldsymbol{\Omega}$ are obtained by cyclic 
interchange of indices), and $\varkappa \approx 10^{-10} \mathrm{eV} \cdot \mathrm{cm}$. Taking matrix elements of $H_{1 \mathrm{~V}}$ and $H_{3 \mathrm{~V}}$ within pairs of the states with $J_{z}= \pm 1 / 2$ (light holes) and $J_{z}= \pm 3 / 2$ (heavy holes), and going to the pseudospin notation, we obtain for a [100] QW:

$$
\begin{aligned}
\mathbf{h}_{l}(\mathbf{k}) & =-\left(2 \frac{\alpha_{V} \hbar^{3}}{m \sqrt{2 m E_{g}}}\left\langle k_{z}^{2}\right\rangle-2 \sqrt{3} \varkappa\right)\left(k_{x} \hat{\jmath}_{x}-k_{y} \hat{\jmath}_{y}\right) \\
\mathbf{h}_{h}(\mathbf{k}) & =-4 \sqrt{3} \varkappa\left(k_{x} \hat{\jmath}_{x}-k_{y} \hat{\jmath}_{y}\right)
\end{aligned}
$$

where $\mathbf{h}_{l}(\mathbf{k})$ and $\mathbf{h}_{h}(\mathbf{k})$ are spin-orbit fields (see Eq.(3)) for light and heavy holes respectively. Consequently, $\gamma_{l}=\left(\frac{4 \alpha_{V} \hbar m_{l}}{m \sqrt{2 m E_{g}}}\left\langle k_{z}^{2}\right\rangle-\frac{4 \sqrt{3} m_{l}}{\hbar^{2}} \varkappa\right) R_{12}$ for light holes, and $\gamma_{h}=$ $\frac{8 \sqrt{3} m_{h}}{\hbar^{2}} \varkappa R_{12}$ for heavy holes, where $m_{l}$ and $m_{h}$ are effective masses of the light and heavy hole respectively, corresponding to their motion along the QW plane. For example, in a 10nm-wide GaAs QW, $\gamma_{l} \approx \gamma_{h} \approx\left(2 \cdot 10^{5} \mathrm{~cm}^{-1}\right) R_{12}$.

The symmetry of exchange interactions has been discussed in relation to feasibility of quantum computation with spins of localized electrons in semiconductor nanostructures [2,4:5]. A necessary (but not sufficient; see Ref. [26]) condition for practical quantum computing to become possible is that the error probability per quantum gate be less than a certain value (of the order of $10^{-5}$ ) [27]. As shown in Ref. [5], there exists a way of performing exchange-mediated quantum gates that allows to avoid errors caused by the anisotropy, provided $\gamma$ remains constant when the isotropic exchange constant is changed. The above consideration shows that this is indeed the case as long as spin-orbit constants do not change. They may change, however, because application of electric fields to the structure can either alter $\left\langle k_{z}^{2}\right\rangle$ or bring about SIA (Rashba) terms. Since typical values of $\gamma$ are in reality much greater than estimated in Ref. [5], the uncontrollable effect of anisotropy on exchange-mediated quantum gates can not be so easily discarded.

In conclusion, the exchange interaction of charge carriers (electrons or holes) localized in two-dimensional semiconductor structures is shown to be described by an universal Hamiltonian in terms of carriers' pseudospins. It has the Heisenberg form unless spin-orbit terms, linear in the carrier wave vector, are present in the total Hamiltonian of the system. In this latter case, anisotropic contributions having both Dzyaloshinskii-Moriya and pseudodipole 
form arise. The "rotation angle" $\gamma$, characterizing the relative strength of the anisotropic exchange, linearly depends on the distance between the localization centers and does not depend on binding energies of the carriers.

The author is grateful to I.A.Merkulov, E.L.Ivchenko, L.P.Gor'kov, and P.G.Krotkov for stimulating discussions. Partial support of INTAS, RFBR, and UK EPSRC is acknowledged. 


\section{REFERENCES}

[1] E.L.Ivchenko, G.E.Pikus, Superlattices and Other Heterostructures, (Springer Verlag, Berlin, 1995).

[2] K.V.Kavokin, Phys.Rev.B 64, 075305 (2001).

[3] I.Dzyaloshinskii, Phys.Chem. Solids 4, 241 (1958); T.Moriya, Phys.Rev. 120, 91 (1960).

[4] N.E.Bonesteel, D.Stepanenko, and D.P.DiVincenzo, Phys.Rev.Lett. 87, 207901 (2001).

[5] G.Burkard and D.Loss, Phys.Rev.Lett. 88, 047903 (2002).

[6] L.-A.Wu, D.A.Lidar, e-print quant-ph/0202168.

[7] R.I.Dzhioev et al, Phys.Rev.B, in press (e-print cond-mat/028083).

[8] L.P.Gor'kov and L.P.Pitaevskii, Dokl.Acad.Nauk SSSR 151, 822 (1963) [Sov.Phys.Dokl. 8, $788(1964)]$

[9] C.Herring and M.Flicker, Phys.Rev. 134, A362 (1964).

[10] L.P.Gor'kov and P.G.Krotkov, e-print cond-mat/0209141.

[11] D.Bimberg, M.Grundmann, and N.N.Ledentsov, Quantum Dot Heterostructures (John Wiley \& Sons, New York (1999)).

[12] M.Brodsky, N.B.Zhitenev, R.C.Ashoori, L.N.Pfeiffer, and K.W.West, Phys.Rev.Lett. 85, $2356(2000)$.

[13] I.V.Ponomarev, V.V.Flambaum, and A.L.Efros, Phys.Rev.B 60, 15848 (1999).

[14] L.D.Landau and E.M.Lifshitz, "Quantum mechanics" (Pergamon Press, New York $(1977))$.

[15] Optical Orientation, edited by F.Meier and B.P.Zakharchenya, Modern Problems in Condensed Matter Sciences Vol.8 (North-Holland, Amsterdam, 1984). 
[16] G.Dresselhaus, Phys.Rev. 100, 580 (1955).

[17] E.I.Rashba, Fiz.Tv.Tela (Leningrad) 2, 1224 (1960) [Sov.Phys.Solid State 2, 1109 (1960)]; Yu.A.Bychkov, E.I.Rashba, Usp.Fiz.Nauk 146, 531 (1985).

[18] B.L.Altshuler et al, Zh.Eksp.Teor.Fiz. 81, 768 (1981) [JETP 54, 411 (1981)].

[19] M.I.Dyakonov, V.Yu.Kachorovskii, $\quad$ Fiz.Techn.Poluprov. $\quad 20, \quad 178$ (1986)[Sov.Phys.Semicond. 20, 110 (1986)]

[20] S.V.Gupalov, E.L.Ivchenko, and A.V.Kavokin, JETP 86, 388 (1998).

[21] L.S.Levitov, E.I.Rashba, e-print cond-mat/0209507.

[22] A.Abragam and B.Bliney, Electron paramagnetic resonance of transition ions (Clarendon Press, Oxford, 1970).

[23] B.Jusserand, D.Richard, G.Allan, C.Priester, and B.Etienne, Phys.Rev.B 51, 4707 (1995)

[24] Z.Willamowski, W.Jantsch, H.Malissa, and U.Rössler, Phys.Rev.B 66, 195315 (2002).

[25] Chapter 3 of Ref. [15] (by G.E.Pikus and A.N.Titkov), and references therein.

[26] M.Dyakonov, e-print cond-mat/0110326.

[27] J.Preskill, Proc.Roy.Soc.London 454, 385 (1998). 